International Journal of Pure and Applied Mathematics

Volume 93 No. 2 2014, 261-274

ISSN: 1311-8080 (printed version); ISSN: 1314-3395 (on-line version)

url: http://www.ijpam.eu

doi: http://dx.doi.org/10.12732/ijpam.v93i2.10

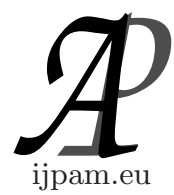

\title{
SOME COMMON FIXED POINT THEOREMS FOR WEAKLY RECIPROCALLY CONTINUOUS MAPPINGS IN A FUZZY METRIC SPACE
}

\author{
Shin Min Kang ${ }^{1 \S}$, Sanjay Kumar ${ }^{2}$, Vishal Gupta ${ }^{3}$, Balbir Singh ${ }^{4}$ \\ ${ }^{1}$ Department of Mathematics and RINS \\ Gyeongsang National University \\ Jinju, 660-701, KOREA \\ ${ }^{2}$ Department of Mathematics \\ Deenbandhu Chhotu Ram University of Science and Technology \\ Murthal, 131039, INDIA \\ ${ }^{3}$ Department of Mathematics \\ Maharishi Markandeshwar University \\ Mullana, 133203, INDIA \\ ${ }^{4}$ Department of Mathematics \\ Bhagwan Mahaveer Institute of Engineering and Technology \\ Sonipat, 131001, INDIA
}

\begin{abstract}
In this paper, we prove some common fixed point theorems for weakly reciprocally continuous mappings satisfying contractive conditions. Also, we prove a common fixed point theorems in a fuzzy metric space from the class of compatible mappings to non-compatible mappings in a fuzzy metric space.
\end{abstract}

AMS Subject Classification: 47H10, 54H25

Key Words: compatible mapping, non-compatible mapping, weakly reciprocally continuous mapping, $R$-weakly commuting mapping, $R$-weakly commuting of type $\left(A_{f}\right)$, of type $\left(A_{g}\right)$ and of type $(P)$

Received: February 28, 2014

(C) 2014 Academic Publications, Ltd. url: www.acadpubl.eu

$\S$ Correspondence author 


\section{Introduction and Preliminaries}

It proved a turning point in the development of fuzzy mathematics when the notion of fuzzy set was introduced by Zadeh [17]. Fuzzy set theory has many applications in applied sciences such as neural network theory, stability theory, mathematical programming, modelling theory, engineering sciences, medical sciences, image processing, control theory and communication, etc. There are many view points of the notion of the metric space in fuzzy topology (see Deng [1], Erceg [2], George and Veermani [3], Kramosil and Michálek [7], Kaleva and Seikkala [8]).

In this paper, we are considering the fuzzy metric space in the sense of Kramosil and Michálek [7].

Definition 1.1. A binary operation $\Delta$ on $[0,1]$ is a $t$-norm if it satisfies the following conditions:

(i) $\Delta$ is associative and commutative,

(ii) $\Delta(a, 1)=a$ for every $a \in[0,1]$,

(iii) $\Delta(a, b) \leq \Delta(c, d)$, whenever $a \leq c$ and $b \leq d$.

Basics examples of $t$-norm are the Lukasiewicz $t$-norm $\Delta_{L}, \Delta_{L}(a, b)=$ $\max \{a+b-1,0\}$, the product $t$-norm $\Delta_{P}, \Delta_{P}(a, b)=a b$ and the minimum $t$-norm $\Delta_{M}$ and $\Delta_{M}(a, b)=\min \{a, b\}$.

Definition 1.2. The 3 -triple $(X, M, \Delta)$ is called a fuzzy metric space if $X$ is an arbitrary set, $\Delta$ is a continuous $t$-norm and $M$ is a fuzzy set on $X^{2} \times[0, \infty)$ satisfying the following conditions: for all $x, y, z \in X$ and $s, t>0$

(FM-1) $M(x, y, 0)=0$,

(FM-2) $M(x, y, t)=1$ for all $t>0$ if and only if $x=y$,

(FM-3) $M(x, y, t)=M(y, x, t)$,

(FM-4) $M(x, z, t+s) \geq \Delta(M(x, y, t), M(y, z, s))$,

$($ FM-5) $M(x, y, \cdot):[0, \infty) \rightarrow[0,1]$ is left continuous.

Note that $M(x, y, t)$ can be thought of as the degree of nearness between $x$ and $y$ with respect to $t$.

Remark 1.3. ([4]) In a fuzzy metric space $(X, M, \Delta), M(x, y, t)$ is nondecreasing with respect to $t$ for all $x, y \in X$.

Definition 1.4. A sequence $\left\{x_{n}\right\}$ in a fuzzy metric space $(X, M, \Delta)$ is said to be

(1) convergent to the limit $x$ if $\lim _{n \rightarrow \infty} M\left(x_{n}, x, t\right)=1$ for all $t>0$,

(2) Cauchy sequence in $X$ if for every $\lambda \in(0,1)$ and $t>0$, there exists a positive integer $N$ such that $M\left(x_{n}, x_{m}, t\right)>1-\lambda$ whenever $m, n \geq N$, 
(3) complete if every Cauchy sequence in $X$ is convergent in $X$.

Fixed point theory in fuzzy metric spaces has been developing since the paper of Grabiec [4]. Subramanyam [15] gave a generalization of Jungck's theorem ([5]) for commuting mapping in the setting of fuzzy metric spaces. In the recent literature of metric fixed point theory, weaker conditions of commutativity are described as: weakly commuting mappings ([14]), compatible mappings ([5]), $R$-weakly commuting mappings ([10]), $R$-weakly commutativity of type $\left(A_{g}\right)$ ([13]) and $R$-weakly commutativity of type maps $\left(A_{f}\right)([13])$. It is to be noted that all such mapping commute at their coincidence points.

In 1999, Vasuki [16] introduced the notion of weakly commuting as follows:

Definition 1.5. Let $f$ and $g$ be self-mappings on a fuzzy metric space $(X, M, \Delta)$. Then $f$ and $g$ are said to be weakly commuting if $M(f g x, g f x, t) \geq$ $M(f x, g x, t)$ for all $x \in X$ and $t>0$.

In 1994, Mishra [9] generalized the notion of weakly commuting to compatible mappings in fuzzy metric spaces akin to the concept of compatible mapping in metric spaces (see Jungck [5]).

Definition 1.6. Let $f$ and $g$ be self-mappings on a fuzzy metric space $(X, M, \Delta)$. Then $f$ and $g$ is said to be compatible if $\lim _{n \rightarrow \infty} M\left(f g x_{n}, g f x_{n}, t\right)=1$ whenever $\left\{x_{n}\right\}$ is a sequence in $X$ such that $\lim _{n \rightarrow \infty} f x_{n}=\lim _{n \rightarrow \infty} g x_{n}=u$ for some $u \in X$ and for all $t>0$.

In 1994, Pant [10] introduced the concept of $R$-weakly commuting mappings in metric spaces. Later on, Vasuki [16] initiated the concept of non-compatible mapping in fuzzy metric spaces and introduced the notion of $R$-weakly commuting mapping in fuzzy metric spaces and proved some common fixed point theorems for these mappings.

Definition 1.7. Let $f$ and $g$ be self-mappings on a fuzzy metric space $(X, M, \Delta)$. Then $f$ and $g$ is said to be non-compatible if $\lim _{n \rightarrow \infty} M\left(f g x_{n}, g f x_{n}, t\right) \neq$ 1 or non-existent whenever $\left\{x_{n}\right\}$ is a sequence in $X$ such that $\lim _{n \rightarrow \infty} f x_{n}=$ $\lim _{n \rightarrow \infty} g x_{n}=u$ for some $u \in X$ and for all $t>0$.

Definition 1.8. Let $f$ and $g$ be self-mappings on a fuzzy metric space $(X, M, \Delta)$. Then $f$ and $g$ is said to be $R$-weakly commuting if there exists $R>0$ such that $M(f g x, g f x, t) \geq M(f x, g x, t / R)$ for all $x \in X$.

Obviously weak commutativity implies $R$-weak commutativity. However, $R$-weak commutativity implies weak commutativity only when $R \leq 1$.

Definition 1.9. ([6]) Let $f$ and $g$ be self-mappings on a fuzzy metric space 
$(X, M, \Delta)$. Then $f$ and $g$ are called, for all $x \in X$ and $t>0$,

(1) $R$-weakly commuting of type $\left(A_{g}\right)$ if there exists $R>0$ such that $M(f f x, g f x, t) \geq M(f x, g x, t / R)$,

(2) $R$-weakly commuting of type $\left(A_{f}\right)$ if there exists some $R>0$ such that $M(f g x, g g x, t) \geq M(f x, g x, t / R)$,

(3) $R$-weakly commuting of type $(P)$ if there exists $R>0$ such that $M(f f x$, $g g x, t) \geq M(f x, g x, t / R)$.

It is obvious that pointwise $R$-weakly commuting mappings commute at their coincidence points and $R$-weak commutativity is equivalent to commutativity at coincidence points. It may be noted that both compatible and noncompatible mappings can be $R$-weakly commuting of the type $\left(A_{g}\right)$ or of type $\left(A_{f}\right)$, but converse need not be true.

In 1999, Pant [11] introduced a new continuity condition, known as reciprocal continuity as follows:

Definition 1.10. Let $f$ and $g$ be self-mappings on a fuzzy metric space $(X, M, \Delta)$. Then $f$ and $g$ are called reciprocally continuous if $\lim _{n \rightarrow \infty} f g x_{n}=$ $f z$ and $\lim _{n \rightarrow \infty} g f x_{n}=g z$, whenever $\left\{x_{n}\right\}$ is a sequence such that $\lim _{n \rightarrow \infty} f x_{n}=$ $\lim _{n \rightarrow \infty} g x_{n}=z$ for some $z \in X$.

If $f$ and $g$ are both continuous, then they are obviously reciprocally continuous, but the converse is need not be true.

Recently, Pant et al. [12] generalized the notion of reciprocal continuity to weak reciprocal continuity as follows:

Definition 1.11. Let $f$ and $g$ be self-mappings on a fuzzy metric space $(X, M, \Delta)$. Then $f$ and $g$ are called weakly reciprocally continuous if $\lim _{n \rightarrow \infty} f g x_{n}=$ $f z$ or $\lim _{n \rightarrow \infty} g f x_{n}=g z$, whenever $\left\{x_{n}\right\}$ is a sequence such that $\lim _{n \rightarrow \infty} f x_{n}=$ $\lim _{n \rightarrow \infty} g x_{n}=z$ for some $z \in X$.

If $f$ and $g$ are reciprocally continuous, then they are obviously weak reciprocally continuous, but the converse is not true.

Now an application of weak reciprocal continuity, we prove common fixed point theorems under contractive conditions. Also we prove common fixed point theorems from the class of compatible mappings to a wider class of mappings which also includes non-compatible mappings in a fuzzy metric space.

Lemma 1.12. ([9]) Let $(X, M, \Delta)$ be a fuzzy metric space. If there exists $q \in(0,1)$ such that $M(x, y, q t) \geq M(x, y, t)$ for all $x, y \in X$ and $t>0$, then $x=y$. 
Lemma 1.13. ([9]) Let $\left\{y_{n}\right\}$ be a sequence in a fuzzy metric space $(X, M, \Delta)$. If there exists $q \in(0,1)$ such that $M\left(y_{n+1}, y_{n}, q t\right) \geq M\left(y_{n}, y_{n-1}, t\right)$ for all $n \in \mathbb{N}$ and $t>0$, then $\left\{y_{n}\right\}$ is a Cauchy sequence in $X$.

\section{Main Theorems}

Now we give some fixed point theorems using weak reciprocal continuity in a fuzzy metric space.

Theorem 2.1. Let $f$ and $g$ be weakly reciprocally continuous self-mappings of a complete fuzzy metric space $(X, M, \Delta)$ with $\Delta(p, p) \geq p$ for all $p \in[0,1]$ satisfying the following conditions:

$$
\begin{gathered}
f(X) \subset g(X), \\
M(f x, f y, q t) \geq \min \{M(g x, g y, t), M(g x, f y, 2 t), M(f x, g x, t), \\
M(f x, g y, t), M(f y, g y, t)\}
\end{gathered}
$$

for any $x, y \in X$ and $t>0$, where $q \in(0,1)$, $(C 3) f$ and $g$ are either compatible or $R$-weakly commuting of type $\left(A_{g}\right)$ or $R$-weakly commuting of type $\left(A_{f}\right)$ or $R$-weakly commuting of the type $(P)$. Then $f$ and $g$ have a unique common fixed point.

Proof. Since $f(X) \subset g(X)$, there exists a sequence $\left\{x_{n}\right\}$ in $X$ such that $f x_{n}=g x_{n+1}$.

Also, we can define a sequence $\left\{y_{n}\right\}$ in $X$ as

$$
y_{n}=f x_{n}=g x_{n+1} .
$$

Now, we show that $\left\{y_{n}\right\}$ is a Cauchy sequence in $X$. By $(C 2)$, we have

$$
\begin{aligned}
M\left(y_{n}, y_{n+1}, q t\right) & \\
= & M\left(f x_{n}, f x_{n+1}, q t\right) \\
\geq & \min \left\{M\left(g x_{n}, g x_{n+1}, t\right), M\left(g x_{n}, f x_{n+1}, 2 t\right), M\left(f x_{n}, g x_{n}, t\right),\right. \\
& \left.M\left(f x_{n}, g x_{n+1}, t\right), M\left(f x_{n+1}, g x_{n+1}, t\right)\right\} \\
= & \min \left\{M\left(y_{n-1}, y_{n}, t\right), M\left(y_{n-1}, y_{n+1}, 2 t\right), M\left(y_{n}, y_{n-1}, t\right),\right. \\
& \left.M\left(y_{n}, y_{n}, t\right), M\left(y_{n+1}, y_{n}, t\right)\right\} \\
\geq & \min \left\{M\left(y_{n-1}, y_{n}, t\right), \Delta\left(M\left(y_{n-1}, y_{n}, t\right), M\left(y_{n}, y_{n+1}, t\right)\right),\right. \\
& \left.M\left(y_{n}, y_{n-1}, t\right), 1, M\left(y_{n+1}, y_{n}, t\right)\right\} \\
= & M\left(y_{n-1}, y_{n}, t\right),
\end{aligned}
$$


By Lemma 1.13, the sequence $\left\{y_{n}\right\}$ is a Cauchy sequence in $X$. Since $X$ is complete, there exists a point $z \in X$ such that $\lim _{n \rightarrow \infty} y_{n}=z$. Therefore, by (2.1), we have

$$
\lim _{n \rightarrow \infty} y_{n}=\lim _{n \rightarrow \infty} f x_{n}=\lim _{n \rightarrow \infty} g x_{n+1}=z .
$$

Suppose that $f$ and $g$ are compatible mappings. Now by weak reciprocal continuity of $f$ and $g$, we have $\lim _{n \rightarrow \infty} f g x_{n}=f z$ or $\lim _{n \rightarrow \infty} g f x_{n}=g z$.

Let $\lim _{n \rightarrow \infty} g f x_{n}=g z$. Then compatibility of $f$ and $g$ gives

$$
\lim _{n \rightarrow \infty} M\left(f g x_{n}, g f x_{n}, t\right)=1
$$

and hence $\lim _{n \rightarrow \infty} f g x_{n}=g z$. By (2.1), we get $\lim _{n \rightarrow \infty} f g x_{n+1}=\lim _{n \rightarrow \infty} f f x_{n}=g z$. Therefore, by $(C 2)$, we get

$$
\begin{gathered}
M\left(f z, f f x_{n}, t\right) \geq \min \left\{M\left(g z, g f x_{n}, t\right), M\left(g z, f f x_{n}, 2 t\right), M(f z, g z, t),\right. \\
\left.M\left(f z, g f x_{n}, t\right), M\left(f f x_{n}, g f x_{n}, t\right)\right\} .
\end{gathered}
$$

Taking limit as $n \rightarrow \infty$, we get

$$
\begin{aligned}
M(f z, g z, q t) \geq \min \{M(g z, g z, t), M(g z, g z, 2 t), M(f z, g z, t), & \\
& M(f z, g z, t), M(g z, g z, t)\} \\
= & M(f z, g z, t) .
\end{aligned}
$$

Thus, by Lemma 1.12, we have $f z=g z$. Again compatibility of $f$ and $g$ implies commutativity at coincidence point. Hence $g f z=f f z=g g z$. Using $(C 2)$, we obtain

$$
\begin{aligned}
M(f z, f f z, q t) \geq \min \{M(g z, g f z, t), M(g z, f f z, 2 t), M(f z, g z, t), & \\
& (f z, g f z, t), M(f f z, g f z, t)\} \\
\geq \min \{M(f z, f f z, t), \Delta(1, M(f z, f f z, t)), 1, & \\
& M(f z, f f z, t), 1\} \\
= & M(f z, f f z, t),
\end{aligned}
$$

that is, we have $f z=f f z$. Hence $f z=f f z=g f z$ and $f z$ is a common fixed point of $f$ and $g$.

Next suppose that $\lim _{n \rightarrow \infty} f g x_{n}=f z$. Then by $f(X) \subset g(X)$, there exists $u \in X$ such that $f z=g u$ and hence $\lim _{n \rightarrow \infty} f g x_{n}=g u$. Compatibility of $f$ and 
$g$ implies $\lim _{n \rightarrow \infty} g f x_{n}=g u$. By (2.1), we have $\lim _{n \rightarrow \infty} f g x_{n+1}=\lim _{n \rightarrow \infty} f f x_{n}=g u$. Therefore, by $(C 2)$, we get

$$
\begin{gathered}
M\left(f u, f f x_{n}, t\right) \geq \min \left\{M\left(g u, g f x_{n}, t\right), M\left(g u, f f x_{n}, 2 t\right), M(f u, g u, t),\right. \\
\left.M\left(f u, g f x_{n}, t\right), M\left(f f x_{n}, g f x_{n}, t\right)\right\} .
\end{gathered}
$$

Taking limit as $n \rightarrow \infty$, we get

$$
\begin{aligned}
M(f u, g u, q t) \geq \min \{ & M(g u, g u, t), M(g u, g u, 2 t), M(f u, g u, t), \\
& M(f u, g u, t), M(g u, g u, t)\} \\
= & M(f u, g u, t),
\end{aligned}
$$

so that, we have $f u=g u$. Again compatibility of $f$ and $g$ implies commutativity at coincidence point. Hence $f g u=g g u=f f u=g f u$. Finally, using $(C 2)$, we obtain

$$
\begin{aligned}
M(f u, f f u, q t) \geq \min \{ & M(g u, g f u, t), M(g u, f f u, 2 t), M(f u, g u, t), \\
& M(f u, g f u, t), M(f f u, g f u, t)\} \\
\geq \min \{M(f u, f f u, t), \Delta(1, M(f u, f f u, t)), 1, & \\
& M(f u, f f u, t), 1\} \\
= & M(f u, f f u, t),
\end{aligned}
$$

that is, we have $f u=f f u$. Hence $f u=g f u$ and $f u$ is a common fixed point of $f$ and $g$.

Now suppose that $f$ and $g$ are $R$-weakly commuting of type $\left(A_{g}\right)$. Now by weak reciprocal continuity of $f$ and $g$, we have $\lim _{n \rightarrow \infty} f g x_{n}=f z$ or $\lim _{n \rightarrow \infty} g f x_{n}=$ $g z$.

Let $\lim _{n \rightarrow \infty} g f x_{n}=g z$. Then $R$-weak commutativity of the type $\left(A_{g}\right)$ of $f$ and $g$ implies that

$$
\lim _{n \rightarrow \infty} M\left(f f x_{n}, g f x_{n}, t\right) \geq \lim _{n \rightarrow \infty} M\left(f x_{n}, g x_{n}, t / R\right)=1
$$

and hence $\lim _{n \rightarrow \infty} f f x_{n}=g z$. Also, using $(C 2)$, we get

$$
\begin{gathered}
M\left(f z, f f x_{n}, t\right) \geq \min \left\{M\left(g z, g f x_{n}, t\right), M\left(g z, f f x_{n}, 2 t\right), M(f z, g z, t),\right. \\
\left.M\left(f z, g f x_{n}, t\right), M\left(f f x_{n}, g f x_{n}, t\right)\right\} .
\end{gathered}
$$

Taking limit as $n \rightarrow \infty$, we get

$$
\begin{aligned}
M(f z, g z, q t) \geq \min \{M(g z, g z, t), M(g z, g z, 2 t), M(f z, g z, t), & M(f z, g z, t), M(g z, g z, t)\} \\
= & M(f z, g z, t),
\end{aligned}
$$


so that, we have $f z=g z$. Again by using $R$-weak commutativity of type $\left(A_{g}\right)$, $M(f f z, g f z, t) \geq M(f z, g z, t / R)=1$. This yields $f f z=g f z$ and hence $f f z=$ $f g z=g f z=g g z$. Using $(C 2)$, we get

$$
\begin{aligned}
M(f z, f f z, q t) \geq \min \{ & M(g z, g f z, t), M(g z, f f z, 2 t), M(f z, g z, t), \\
& M(f z, f f z, t), M(f f z, g f z, t)\} \\
= & \min \{M(f z, f f z, t), \Delta(1, M(f z, f f z, t)), 1, \\
& M(f z, f f z, t), 1\} \\
= & M(f z, f f z, t),
\end{aligned}
$$

that is, we have $z=f f z$ and hence $f z=f f z=g f z$, Therefore $f z$ is a common fixed point of $f$ and $g$. Similarly, if $\lim _{n \rightarrow \infty} f g x_{n}=f z$, then we can prove easily.

Suppose that $f$ and $g$ are $R$-weakly commuting of type $\left(A_{f}\right)$. Again, as done above, we can easily prove that $f z$ is a common fixed point of $f$ and $g$.

Now suppose that $f$ and $g$ are $R$-weakly commuting of type $(P)$. Now by weak reciprocal continuity of $f$ and $g$, we have $\lim _{n \rightarrow \infty} f g x_{n}=f z$ or $\lim _{n \rightarrow \infty} g f x_{n}=$ $g z$.

Let $\lim _{n \rightarrow \infty} g f x_{n}=g z$. Then by $R$-weak commutativity of the type $(P)$ of $f$ and $g$, we have $M\left(f f x_{n}, g g x_{n}, t\right) \geq M\left(f x_{n}, g x_{n}, t / R\right)$ and hence

$$
\lim _{n \rightarrow \infty} M\left(f f x_{n}, g g x_{n}, t\right) \geq M(z, z, t / R)=1 .
$$

By (2.1), we have $g f x_{n-1}=g g x_{n} \rightarrow g z$ and $f f x_{n} \rightarrow g z$ as $n \rightarrow \infty$. Also, using $(C 2)$, we get

$$
\begin{gathered}
M\left(f z, f f x_{n}, t\right) \geq \min \left\{M\left(g z, g f x_{n}, t\right), M\left(g z, f f x_{n}, 2 t\right), M(f z, g z, t),\right. \\
\left.M\left(f z, g f x_{n}, t\right), M\left(f f x_{n}, g f x_{n}, t\right)\right\} .
\end{gathered}
$$

Taking limit as $n \rightarrow \infty$, we get

$$
\begin{aligned}
& M(f z, g z, q t) \geq \min \{M(g z, g z, t), M(g z, g z, 2 t), M(f z, g z, t), \\
&M(f z, g z, t), M(g z, g z, t)\} \\
&= M(f z, g z, t),
\end{aligned}
$$

so that, we have $f z=g z$. Again by using $R$-weak commutativity of type $(P)$, $M(f f z, g g z, t) \geq M(f z, g z, t / R)=1$. This yields $f f z=g g z$. Hence $f f z=$ 
$f g z=g f z=g g z$. Using $(C 2)$, we get

$$
\begin{aligned}
M(f z, f f z, q t) \geq \min \{M(g z, g f z, t), M(g z, f f z, 2 t), M(f z, g z, t), & \\
& M(f z, g f z, t), M(f f z, g f z, t)\} \\
\geq \min \{M(f z, f f z, t), \Delta(1, M(f z, f f z, t)), 1, & \\
& M(f z, f f z, t), 1\} \\
= & M(f z, f f z, t),
\end{aligned}
$$

that is, $f z=f f z$. Hence $f z=f f z=g f z$ and $f z$ is a common fixed point of $f$ and $g$. Similarly, if $\lim _{n \rightarrow \infty} f g x_{n}=f z$, then we can easily prove.

Finally, in order to prove the uniqueness, let $f w(f z \neq f w)$ be another fixed point of $f$ and $g$. Then, for all $t>0$, we have

$$
\begin{aligned}
& M(f z, f w, q t) \\
& =M(f f z, f f w, q t) \\
& \geq \min \{M(g f z, g f w, t), M(g f z, f f w, 2 t), M(f f z, g f z, t), \\
& \quad M(f f z, g f w, t), M(f f w, g f w, t)\} \\
& \geq \min \{M(f z, f w, t), \Delta(1, M(f z, f w, t)), M(f z, f z, t), \\
& \quad M(f z, f w, t), M(f w, f w, t)\} \\
& =\quad M(f z, f w, t) .
\end{aligned}
$$

Thus we have $f z=f w$. Hence $f z$ is a unique common fixed point of $f$ and $g$. This completes the proof.

We now give an example to illustrate Theorem 2.1.

Example 2.2. Let $X=[2,20]$ and define

$$
M(x, y, t)=\frac{t}{t+|x-y|}
$$

for each $x, y \in X$ and $t>0$. Then $(X, M, \Delta)$ is a complete fuzzy metric space. Define $f, g: X \rightarrow X$ by

$$
f x=\left\{\begin{array}{ll}
2 & \text { if } x=2 \text { or } x>5, \\
6 & \text { if } x<x \leq 5,
\end{array} \quad g x= \begin{cases}2 & \text { if } x=2 \\
11 & \text { if } x<x \leq 5, \\
\frac{x+1}{3} & \text { if } x>5\end{cases}\right.
$$

Let $\left\{x_{n}\right\}$ be sequence in $X$ such that either $x_{n}=2$ or $x_{n}=5+\frac{1}{n}$ for each $n$. Then, clearly, $f$ and $g$ satisfy all the conditions of Theorem 2.1 and have a unique common fixed point at $x=2$. 
Theorem 2.3. Let $f$ and $g$ be weakly reciprocally continuous noncompatible self-mappings of a fuzzy metric space $(X, M, \Delta)$ satisfying $(C 1)$ and the following conditions:

$$
M(f x, f y, q t) \geq M(g x, g y, t)
$$

for any $x, y \in X$,

$$
\begin{aligned}
& M(f x, f f x, q t) \\
& \geq \min \{M(g x, g f x, t), M(f x, g x, 2 t), M(f f x, g f x, t), \\
& \quad M(f x, g f x, t), M(g x, f f x, t)\}
\end{aligned}
$$

for any $x \in X$ and $t>0$, where $q \in(0,1)$,

$(C 6) f$ and $g$ are either $R$-weakly commuting of type $\left(A_{g}\right)$ or $R$-weakly commuting of type $\left(A_{f}\right)$ or $R$-weakly commuting of the type $(P)$.

Then $f$ and $g$ have a common fixed point.

Proof. Since $f$ and $g$ are non-compatible mappings, there exists a sequence $\left\{x_{n}\right\}$ in $X$ such that $\lim _{n \rightarrow \infty} f x_{n}=\lim _{n \rightarrow \infty} g x_{n}=z$ for some $z \in X$, but either $\lim _{n \rightarrow \infty} M\left(f g x_{n}, g f x_{n}, t\right) \neq 1$ or the limit does not exist. Since $f(X) \subset g(X)$ for each $\left\{x_{n}\right\}$, there exists $\left\{y_{n}\right\}$ in $X$ such that $f x_{n}=g y_{n}$. Thus

$$
\lim _{n \rightarrow \infty} f x_{n}=\lim _{n \rightarrow \infty} g x_{n}=\lim _{n \rightarrow \infty} g y_{n}=z .
$$

By virtue of this and using $(C 4)$, we obtain

$$
\lim _{n \rightarrow \infty} M\left(f x_{n}, f y_{n}, q t\right) \geq \lim _{n \rightarrow \infty} M\left(g x_{n}, g y_{n}\right)=1
$$

and hence $\lim _{n \rightarrow \infty} f y_{n}=z$. Therefore, we have

$$
\lim _{n \rightarrow \infty} f x_{n}=\lim _{n \rightarrow \infty} f y_{n}=\lim _{n \rightarrow \infty} g x_{n}=\lim _{n \rightarrow \infty} g y_{n}=z .
$$

Suppose that $f$ and $g$ are $R$-weakly commuting of type $\left(A_{g}\right)$. Then by weak reciprocal continuity of $f$ and $g$, we have $\lim _{n \rightarrow \infty} f g x_{n}=f z$ or $\lim _{n \rightarrow \infty} g f x_{n}=g z$.

Let $\lim _{n \rightarrow \infty} g f x_{n}=g z$. Then $R$-weak commutativity of the type $\left(A_{g}\right)$ of $f$ and $g$ yields

$$
\lim _{n \rightarrow \infty} M\left(f f x_{n}, g f x_{n}, t\right) \geq \lim _{n \rightarrow \infty} M\left(f x_{n}, g x_{n}, t / R\right)=1
$$

and hence $\lim _{n \rightarrow \infty} f f x_{n}=g z$. 
Also, using $(C 4)$, we get $M\left(f f x_{n}, f z, q t\right) \geq M\left(g f x_{n}, g z, t\right)$. Taking limit as $n \rightarrow \infty$, we get

$$
M(g z, f z, q t) \geq M(g z, g z, t)=1 .
$$

Thus we have $f z=g z$. Again by using $R$-weak commutativity of type $\left(A_{g}\right)$, $M(f f z, g f z, t) \geq M(f z, g z, t / R)=1$. This yields $f f z=g f z$ and hence $f f z=$ $f g z=g f z=g g z$. By using $(C 5)$, we get

$$
\begin{aligned}
& M(f z, f f z, q t) \\
& \geq \min \{M(g z, g f z, t), M(f z, g z, 2 t), M(f f z, g f z, t), \\
&M(f z, f f z, t), M(f z, f f z, t)\} \\
&= \min \{M(f z, f f z, t), 1,1, M(f z, f f z, t), M(f z, f f z, t)\} \\
&= M(f z, f f z, t) .
\end{aligned}
$$

Hence, by Lemma 1.12, $f z=f f z=f f z=g f z$ and $f z$ is a common fixed point of $f$ and $g$. Similarly, we prove if $\lim _{n \rightarrow \infty} f g x_{n}=f z$, then again $f z$ is a common fixed point of $f$ and $g$.

The proof is similar if $f$ and $g$ are $R$-weakly commuting of type $\left(A_{f}\right)$ or type $(P)$. This completes the proof.

We now give an example to illustrate Theorem 2.3.

Example 2.4. ([6]) Let $(X, M, \Delta)$ be a fuzzy metric space and $f, g: X \rightarrow$ $X$ as defined in Example 2.2.

Let $\left\{x_{n}\right\}$ be sequence in $X$ such that either $x_{n}=2$ or $x_{n}=5+\frac{1}{n}$ for each $n$. Then, clearly, $f$ and $g$ satisfy all the conditions of Theorem 2.3 and have a common fixed point at $x=2$.

Theorem 2.5. Let $f$ and $g$ be weakly reciprocally continuous noncompatible self-mappings of a fuzzy metric space $(X, M, \Delta)$ satisfying $(C 1)$, $(C 4),(C 6)$ and the following condition:

$$
M(f x, f f x, t) \geq M(g x, g g x, t),
$$

whenever $f x \neq f f x$ for all $x \in X$ and $t>0$.

Then $f$ and $g$ have a common fixed point.

Proof. Since $f$ and $g$ are non-compatible mappings, there exists a sequence $\left\{x_{n}\right\}$ in $X$ such that $\lim _{n \rightarrow \infty} f x_{n}=\lim _{n \rightarrow \infty} g x_{n}=z$ for some $z \in X$, but either $\lim _{n \rightarrow \infty} M\left(f g x_{n}, g f x_{n}, t\right) \neq 1$ or the limit does not exist. Since $f(X) \subset g(X)$ for each $\left\{x_{n}\right\}$, there exists $\left\{y_{n}\right\}$ in $X$ such that $f x_{n}=g y_{n}$. Thus

$$
\lim _{n \rightarrow \infty} f x_{n}=\lim _{n \rightarrow \infty} g x_{n}=\lim _{n \rightarrow \infty} g y_{n}=z .
$$


By virtue of this and using $(C 4)$, we obtain

$$
\lim _{n \rightarrow \infty} M\left(f x_{n}, f y_{n}, q t\right) \geq \lim _{n \rightarrow \infty} M\left(g x_{n}, g y_{n}, t\right)=1 .
$$

This gives $\lim _{n \rightarrow \infty} f y_{n}=z$. Therefore, we have

$$
\lim _{n \rightarrow \infty} f y_{n}=\lim _{n \rightarrow \infty} g x_{n}=\lim _{n \rightarrow \infty} g y_{n}=z .
$$

Suppose that $f$ and $g$ are $R$-weakly commuting of type $\left(A_{g}\right)$. Then by weak reciprocal continuity of $f$ and $g$, we have $\lim _{n \rightarrow \infty} f g x_{n}=f z$ or $\lim _{n \rightarrow \infty} g f x_{n}=g z$.

Let $\lim _{n \rightarrow \infty} g f x_{n}=g z$. Then $R$-weak commutativity of the type $\left(A_{g}\right)$ of $f$ and $g$ yields

$$
\lim _{n \rightarrow \infty} M\left(f f x_{n}, g f x_{n}, t\right) \geq \lim _{n \rightarrow \infty} M\left(f x_{n}, g x_{n}, t / R\right)=1
$$

and hence $\lim _{n \rightarrow \infty} f f x_{n}=g z$. Also, using $(C 4)$, we get $M\left(f f x_{n}, f z, q t\right) \geq$ $M\left(g f x_{n}, g z, t\right)$. Taking limit as $n \rightarrow \infty$, we get

$$
M(g z, f z, q t) \geq M(g z, g z, t)=1 .
$$

Thus by Lemma 1.12, we have $f z=g z$. Again by using $R$-weak commutativity of type $\left(A_{g}\right), M(f f z, g f z, t) \geq M(f z, g z, t / R)=1$. This yields $f f z=g f z$. and hence $f f z=f g z=g f z=g g z$. By using $(C 7)$, we get

$$
M(f z, f f z, t) \geq M(g z, g g z, t)=M(f z, f f z, t),
$$

which is a contraction. Hence $f z=f f z=g f z$ and $f z$ is a common fixed point of $f$ and $g$. Similarly, we can prove if $\lim _{n \rightarrow \infty} f g x_{n}=f z$, then again $f z$ is a common fixed point of $f$ and $g$.

The proof is similar if $f$ and $g$ are $R$-weakly commuting of type $\left(A_{f}\right)$ or of type $(P)$. This complets the proof.

We now give an example to illustrate Theorem 2.5.

Example 2.6. Let $(X, M, \Delta)$ is a fuzzy metric space as defined in Example 2.1. Define $f, g: X \rightarrow X$ by

$$
f x=\left\{\begin{array}{ll}
2 & \text { if } x=2 \text { or } x>5, \\
4 & \text { if } x<x \leq 5,
\end{array} \quad g x= \begin{cases}2 & \text { if } x=2, \\
4 & \text { if } 2<x \leq 5, \\
\frac{x+1}{3} & \text { if } x>5 .\end{cases}\right.
$$

Let $\left\{x_{n}\right\}$ be sequence in $X$ such that either $x_{n}=2$ or $x_{n}=5+\frac{1}{n}$ for each $n$. Then, clearly, $f$ and $g$ satisfy all the conditions of Theorem 2.5 and have common fixed points at $x=2$ and $x=4$. 


\section{Acknowledgments}

One of authors (Professor Sanjay Kumar) is thankful to University Grants Comission for providing Major Research project under Ref. No. 39-41/2010(SR).

\section{References}

[1] Z. Deng, Fuzzy pseudo-metric space, J. Math. Anal. Appl., 86 (1982), 74-95, doi: 10.1016/0022-247X(82)90255-4

[2] M.A. Erceg, Metric space in fuzzy set theory, J. Math. Anal. Appl., 69 (1979), 205-230, doi: 10.1016/0022-247X(79)90189-6

[3] A. George, P. Veeramani, On some results in fuzzy metric spaces, Fuzzy Sets and Systems, 64 (1994), 395-399, doi: 10.1016/0165-0114(94)90162-7

[4] M. Grabiec, Fixed points in fuzzy metric space, Fuzzy Sets and Systems, 27 (1988), 385-389, doi: 10.1016/0165-0114(88)90064-4

[5] G. Jungck, Compatible mappings and common fixed points, Int. J. Math. Math. Sci., 9 (1986), 771-779, doi: 10.1155/S0161171286000935

[6] M. Imdad, J. Ali, Some common fixed point theorems in fuzzy metric spaces, Math. Commun., 11 (2006), 153-163.

[7] I. Kramosil, J. Michálek, Fuzzy metric and statistical metric spaces, Kybernetika, 11 (1975), 336-344.

[8] O. Kaleva, S. Seikkala, On fuzzy metric spaces, Fuzzy Sets and Systems 12 (1984), 215-229, doi: 10.1016/0165-0114(84)90069-1

[9] S.N. Mishra, N. Sharma, S.L. Singh, Common fixed points of maps on fuzzy metric spaces, Int. J. Math. Math. Sci., 17 (1994), 253-258, doi: $10.1155 / \mathrm{S} 0161171294000372$

[10] R.P. Pant, Common fixed points for noncommuting mapping, J. Math. Anal. Appl., 188 (1994), 436-440, doi: 10.1006/jmaa.1994.1437

[11] R.P. Pant, A Common fixed point theorem under a new condition, Indian J. Pure Appl. Math., 30 (1999), 147-152. 
[12] R.P. Pant, R.K. Bisht, D. Arora, Weak reciprocal continuity and fixed point theorems, Ann. Univ. Ferrara, 57 (2011), 181-190, doi: 10.1007/s11565-011-0119-3

[13] H.K. Pathak, Y.J. Cho, S.M. Kang, Remarks on R-weakly commuting mappings and common fixed point theorems, Bull. Korean Math. Soc., 34 (1997), 247-257.

[14] S. Sessa, On a weak commutativity of mappings in fixed point considerations, Publ. Inst. Math., 32(46) (1982), 149-153.

[15] P.V. Subramanyam, Common fixed point theorems in fuzzy metric spaces, Inform. Sci., 83 (1995), 109-112, doi: 10.1016/0020-0255(94)00043-B

[16] R. Vasuki, Common fixed points for $R$-weakly commuting maps in fuzzy metric spaces Indian J. Pure Appl. Math., 30 (1999), 419-423.

[17] L.A. Zadeh, Fuzzy sets, Inform. and Control, 8 (1965), 338-353. 